\title{
UN DETECTEUR SOLIDE DE TRACES : LE TRIACETATE DE CELLULOSE
}

\author{
J.C. VAREILLE et J.L. TEYSSIER* \\ (Manuscrit reçu le 9 octobre 1972)
}

\begin{abstract}
RÉSUMÉ
Le triacétate de cellulose (Bayer triafol T.N.) est étudié comme détecteur visuel solide de traces de particules chargées (particules $\alpha$, fragments de fission du californium, ions néon, argon, krypton et azote). On montre que :

- Le rapport d'attaque $R_{T}$ augmente lorsque la température diminue et lorsque la normalité de l'agent chimique croît. La présence de plastifiant dans le matériau détecteur accroît $R_{T}$ d'un facteur 2,5 .

- L'énergie d'activation de la réaction "potasse-zone endommagée» par le passage de la particule chargée augmente quand le transfert linéique d'énergie diminue.

- L'énergie maximale d'enregistrement est de $4 \mathrm{MeV}$ pour les particules $\alpha$ et un développement par la potasse 4 fois normale à $60^{\circ} \mathrm{C}$.

Enfin, le détecteur est étalonné pour discriminer en énergie les particules $\alpha$ avec une résolution de $500 \mathrm{keV}$.

\section{ABSTRACT}

Cellulose triacetate (Bayer triafol T.N.) is studied as solid state track detectors of charged particles (alpha particles, fission fragments of californium and accelerated heavy ions : argon, neon, krypton and nitrogen). We prove that :

- Etch rate ratio $R_{T}$ increases when temperature of the etchant is decreased and when the concentration of the etchant is increased.

- As energy loss is increased activation energy for track etching decreases. - Critical energy is $4 \mathrm{MeV}$ for alpha particles when the etching reagent is $\mathrm{KOH}$, ${ }_{4} \mathrm{~N}, 60^{\circ} \mathrm{C}$.

Discrimination of alpha particles of different energies is made by this detector with $500 \mathrm{keV}$ resolution.
\end{abstract}

Plusieurs articles, parus dans cette revue $[1,2,3]$ ont attiré l'attention sur les qualités des détecteurs solides de traces et sur leurs possibilités d'emploi dans des disciplines très différentes (dosimétrie neutronique, géochronologie, cristallographie, ....).

Nous donnons ici un certain nombre de renseignements sur l'un de ces détecteurs. Il s'agit du triacétate de cellulose. Parmi les composés cellulosiques, nous l'avons préféré au nitrate qui a fait et fait encore l'objet de très nombreuses recher-

* Laboratoire des Radiations Ionisantes de l'Université de Limoges, I23, rue A.-Thomas, 87100 Limoges.

RADIOPROTECTION, VOL. 7 - $\mathrm{N}^{\mathrm{O}} 4$ 
ches; quant au diacétate, sa faible sensibilité le rend peu commode d'emploi. Nous avons étudié un triacétate de cellulose sans plastifiant qui est commercialisé par la Société Bayer sous l'appellation triafol T.N.

Nous avons déterminé deux paramètres qui nous paraissent bien caractériser le matériau du point de vue de la détection :

- le rapport d'attaque $R_{T}$,

- l'énergie maximale d'enregistrement $E_{M}$.

Nous avons employé la potasse comme agent chimique et les particules $\alpha$, les fragments de fission du Californium 252 , les ions argon, krypton, azote et néon pour irradier le détecteur.

\section{I - LE RAPPORT D'ATTAQUE $R_{T}$}

Il est défini comme le rapport de la vitesse moyenne d'attaque le long de la trace, $\bar{V}_{T}$, et de la vitesse moyenne d'attaque du matériau non irradié, $\left(\bar{V}_{g}\right)$. En dépit de son caractère macroscopique, il fournit des renseignements intéressants puisqu'il dépend à la fois des conditions de l'attaque chimique, de la nature du matériau et de la particule incidente.

I - MÉthodes de mesure de $\mathrm{R}_{T}$

Nous en avons utilisé trois. Les deux premières reposent sur le fait que $R_{T}$ peut être relié à l'angle limite d'enregistrement $\theta_{L}$, valeur que l'angle d'incidence de la particule ne doit pas dépasser pour que la trace soit révélée. On a :

$$
\cos \theta_{L}=\frac{\mathrm{I}}{\mathrm{R}_{T}}+\frac{p_{m}}{L_{M}}
$$

où $p_{m}$ est la profondeur de champ du microscope; $L_{M}$ est le parcours de la particule incidente dans le matériau.

Lorsque, comme c'est souvent le cas $L_{M} \gg p_{m}$,

$$
\cos \theta_{L} \approx \frac{\mathrm{I}}{\mathrm{R}_{T}}
$$

On a d'abord déterminé $\theta_{L}$ en étudiant les variations de l'efficacité de détection $\eta$ en fonction de l'angle d'incidence $\theta$ de la particule incidente repéré par rapport à la normale à la surface du détecteur. $\eta$ est défini comme le rapport des densités des traces $D$ et $D_{0}$ correspondant respectivement aux angles d'incidence $\theta$ et $\mathrm{o}$. La figure I donne le résultat obtenu pour les particules $\alpha$ d'énergie $3 \mathrm{MeV}$ et une solution de potasse 4 fois normale à $60^{\circ} \mathrm{C}$. De manière arbitraire, on prend comme valeur de $\theta_{L}$ l'angle correspondant à une efficacité de $50 \%$, soit :

$$
\left(\theta_{L}\right)_{\alpha}=72^{0} \pm 2^{0} \quad \text { ou } \quad\left(R_{T}\right)_{\alpha}=3,2 \pm 0,3 .
$$


Dans son principe, la méthode n'a aucune limite; dans la pratique, au fur et à mesure que $\theta$ croît, $D$ diminue; cela peut obliger à des durées d'irradiation prohibitives. Aussi, dans les conditions où nous opérons, ne peut-on espérer mesurer correctement des valeurs de $R_{T}$ supérieures à 14,5 .

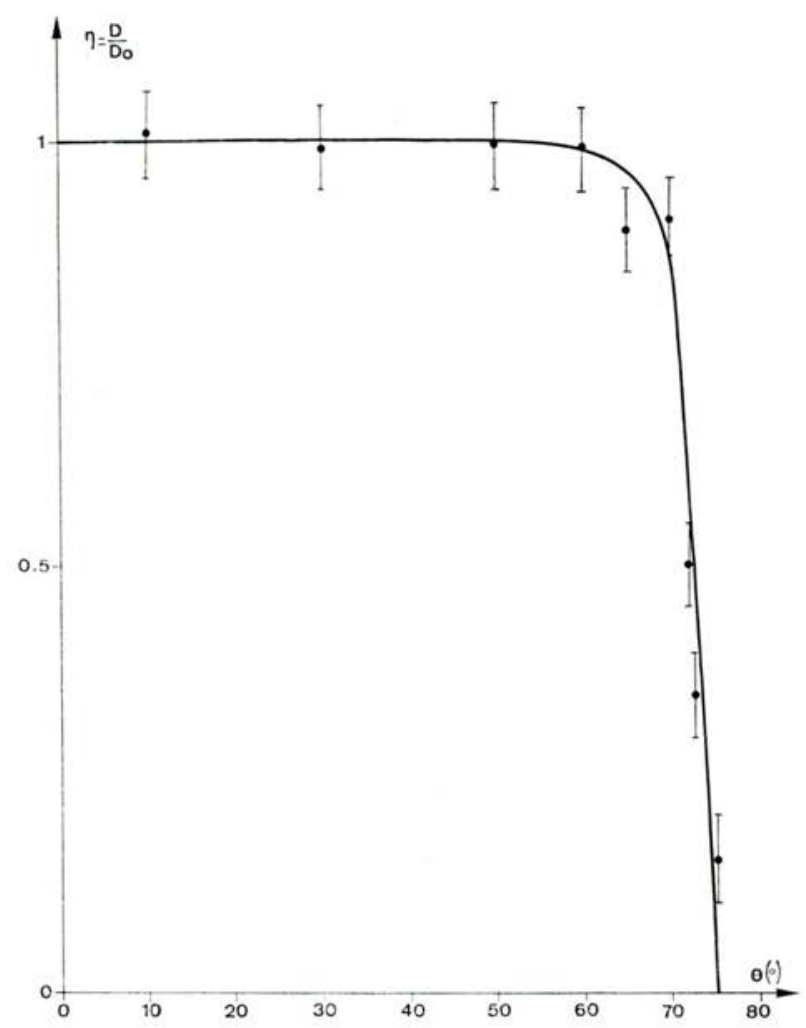

FIG. I. - Efficacité de détection des particules $\alpha$ en fonction de l'angle d'incidence sur le détecteur.

On a également irradié le détecteur sous toutes les incidences possibles à l'aide d'une source de particules quasi ponctuelle obtenue en utilisant un diaphragme de $\mathrm{I} \mathrm{mm}$ de diamètre et de $20 \mu \mathrm{m}$ d'épaisseur. La zone irradiée sur la surface du détecteur situé à la distance $l$ du point source est délimitée par un cercle de rayon $\varrho=l \operatorname{tg} \theta_{L}$.

La mesure de $\varrho$ fournit la valeur de $\theta_{L}$. Dans les mêmes conditions que précédemment, nous avons obtenu :

$$
\left(R_{T}\right)_{\alpha}=2,7 \pm 0,3 .
$$

A cause de l'épaisseur non négligeable du diaphragme, la valeur maximale de $R_{T}$ que l'on peut mesurer est de 3,9.

vOL. $7-\mathrm{N}^{\circ} 4$ 
La dernière méthode est plus longue, mais d'une application beaucoup plus large : on détermine séparément $\bar{V}_{g}$ et $\bar{V}_{T}$ :

- mesure de $\bar{\nabla}_{g}$.

Le matériau présente une face lisse pratiquement exempte de défauts que nous avons choisie pour visualiser les traces; l'autre face est ornée de multiples défauts en formes d'algues. On doit donc s'attendre à une certaine inhomogé-

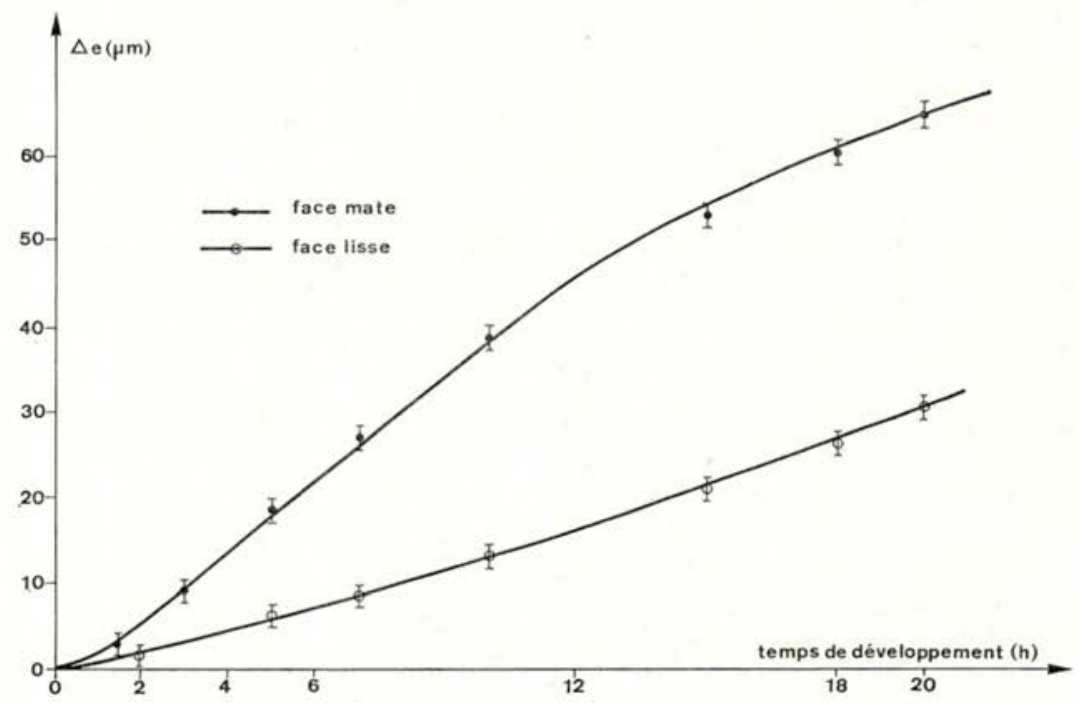

Fig. 2. - Variation d'épaisseur sur chaque face de la feuille de triafol en fonction du temps de développement dans une solution $\mathrm{KOH}(4 \mathrm{~N})$ à $60^{\circ} \mathrm{C}$.

néité du matériau. C'est ce que confirme la diminution d'épaisseur $\overline{\Delta e}$ du matériau pour chaque face en fonction de la durée de l'attaque chimique $t$ (fig. 2). Cependant, comme les pentes des 2 droites sont pratiquement égales pour des temps de développement suffisamment élevés, le matériau est sans doute plus homogène en profondeur.

$\overline{\Delta e}$ est mesuré au comparateur avec une précision de $\mathrm{x} \mu \mathrm{m}$. La vitesse générale moyenne d'attaque $\bar{V}_{g}$ relative à chaque face est définie comme la pente de la partie linéaire de la courbe $\overline{\Delta e}(t)$ correspondante.

$$
\text { - mesure de } \bar{V}_{T}
$$

Nous donnerons ici les résultats obtenus pour les fragments de fission du californium 2 25, car leur taux d'attaque ne peut être déterminé que par l'intermédiaire de $\bar{V}_{g}$ et de $\bar{V}_{T}$. On détermine la loi de variation de la longueur $L$ d'une trace en fonction de $t$. Par l'expérience on a accès aux longueurs $L_{p}$ des 
traces projetées sur la surface; à condition d'opérer sous une incidence donnée, fixée ici à $45^{\circ}$, on peut relier $L_{p}$ et $L$ de manière simple :

$$
L(t)=L_{p}(t) \sqrt{2}+\bar{V}_{g} \sqrt{2} t \quad(t: \text { en heures })
$$

En fait, on prend en compte la valeur moyenne $L_{p}$ des $L_{p}$, puis $\bar{L}$ des $L$ sur roo traces pour que la précision statistique soit bonne.

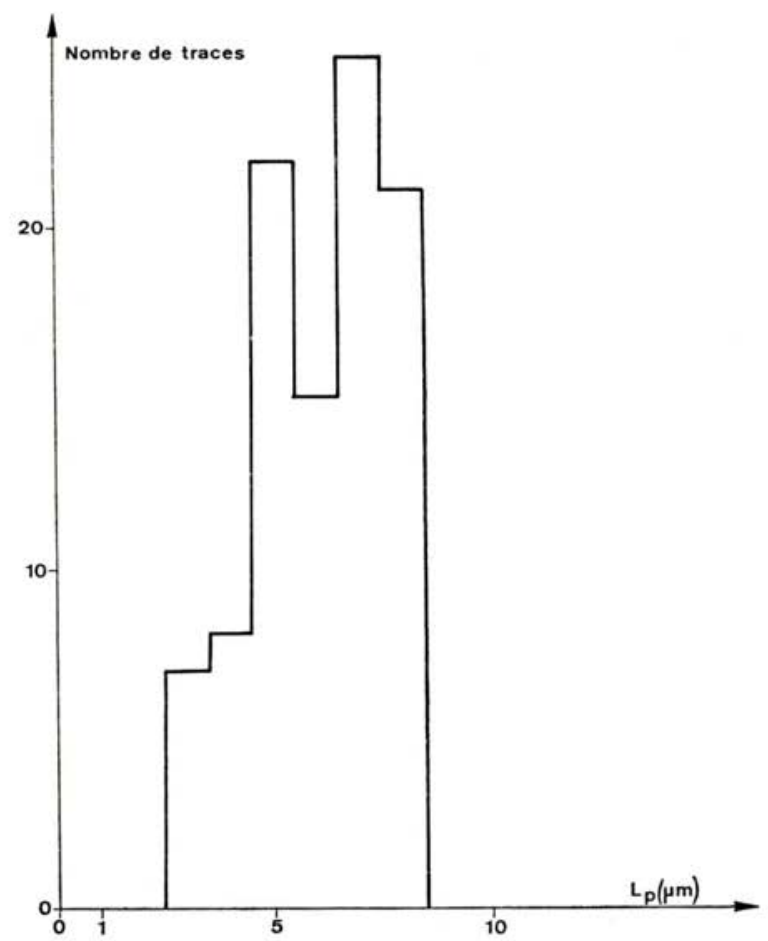

FIg. 3. - Histogramme des longueurs $L_{p}$ des traces des fragments de fission légers pour un développement dans une solution de $\mathrm{KOH}(4 N)$ à $60^{\circ} \mathrm{C}$.

A titre d'exemple, on trouvera sur la figure 3 la répartition statistique de $L_{p}$ pour $t=\mathrm{I} 2 \mathrm{mn}$. Elle rend bien compte de la distribution énergétique des fragments de fission puisque ceux-ci sont de deux sortes (légers et lourds). La courbe $\bar{L}(t)$ (fig. 4) présente une partie linéaire, puis un palier qui correspond à la révélation totale de la trace. La vitesse d'attaque $\bar{V}_{T}$ est définie comme la pente de la partie linéaire. Nous avons consigné dans le tableau I les valeurs de $\bar{V}_{g}, \bar{V}_{T}$ et $\mathrm{R}_{T}$ obtenues avec une solution 4 fois normale de potasse à $60^{\circ} \mathrm{C}$ pour les fragments de fission légers du Californium 252 et les particules $\alpha$ de ${ }_{3} \mathrm{MeV}$.

vol. $7-\mathrm{N}^{\circ} 4$ 
TABLEAU I

Quelques valeurs de $\bar{V}_{g}, \bar{V}_{T}$ et $\mathrm{R}_{T}\left(\mathrm{KOH},{ }_{4} \mathrm{~N}, 60^{\circ} \mathrm{C}\right)$

\begin{tabular}{|c|c|c|c|}
\hline $\begin{array}{c}\bar{V}_{g} \\
(\mu \mathrm{m} / \mathrm{h})\end{array}$ & $\begin{array}{l}\text { Particules } \\
\text { incidentes }\end{array}$ & $\begin{array}{c}\bar{V}_{T} \\
(\mu \mathrm{m} / \mathrm{h})\end{array}$ & $\mathrm{R}_{T}$ \\
\hline \multirow{2}{*}{1,45} & Fragments fission légers & 45,5 & $3 \mathrm{I}$ \\
\hline & $\alpha(\mathrm{MeV})$ & 4,3 & 3 \\
\hline
\end{tabular}

On notera la bonne concordance des valeurs de $\left(R_{T}\right)_{\alpha}$ fournies par les trois méthodes : 3,2, 2,7 et 3 .

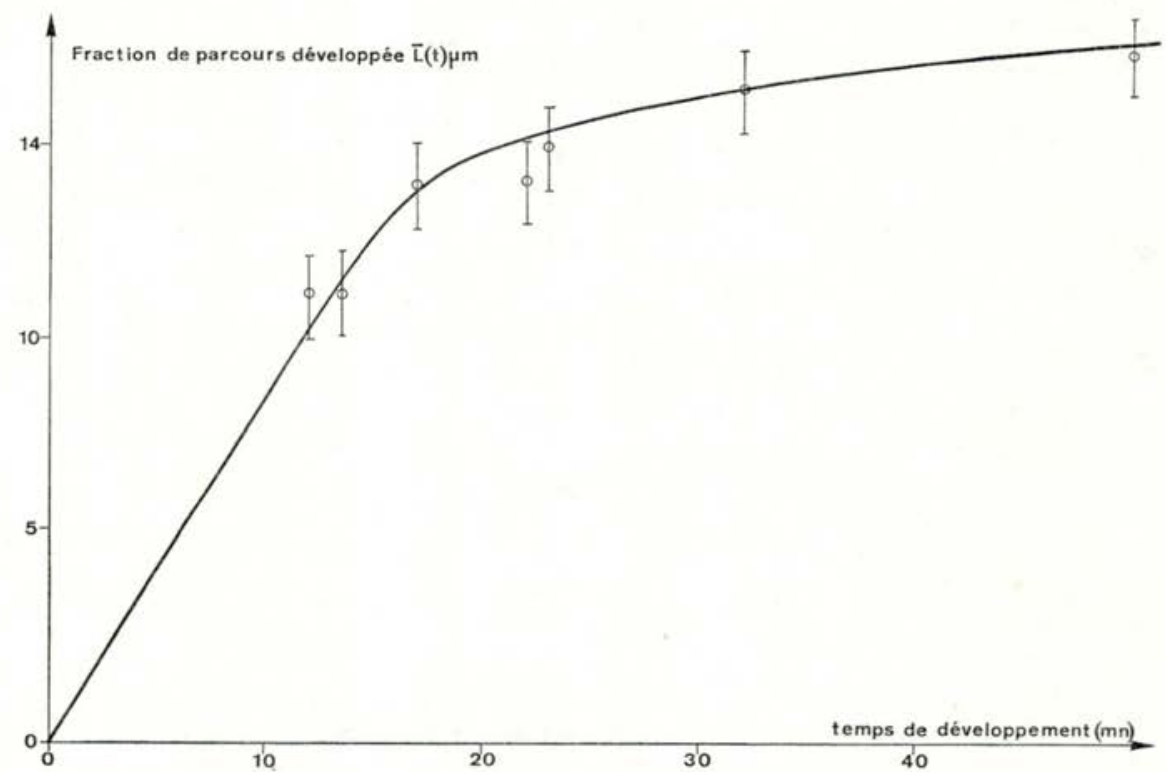

Fig. 4. - Courbe $\bar{L}(t)$ pour les fragments de fission légers dans une solution de $\mathrm{KOH}\left({ }_{4} N\right)$ à $60^{\circ} \mathrm{C}$.

2 - Le rôle de la particule incidente

Nous avons dans des conditions d'attaque déterminées (solution de $\mathrm{KOH}$, $4 \mathrm{~N}$, à $\left.60^{\circ} \mathrm{C}\right)$, mesuré le rapport $R_{T}$ pour :

- des particules $\alpha$ de $3 \mathrm{MeV}$,

- des ions azote de $15,4 \mathrm{MeV}$,

- des ions néon de $22 \mathrm{MeV}$,

- des ions argon de $44 \mathrm{MeV}$,

- des ions krypton de $92 \mathrm{MeV}$,

- des fragments de fission légers du ${ }^{252} \mathrm{Cf}$ (d'énergie moyenne roo $\mathrm{MeV}$ ). 
On caractérise ces particules par leur transfert linéique d'énergie $\frac{\mathrm{d} E}{\mathrm{~d} x}$. En fonction de ce paramètre, $R_{T}$ croît d'abord relativement vite, puis semble tendre vers une limite (fig. 5); des constatations de ce genre ont été faites à plusieurs reprises [5] [7] [8] [9] [10].

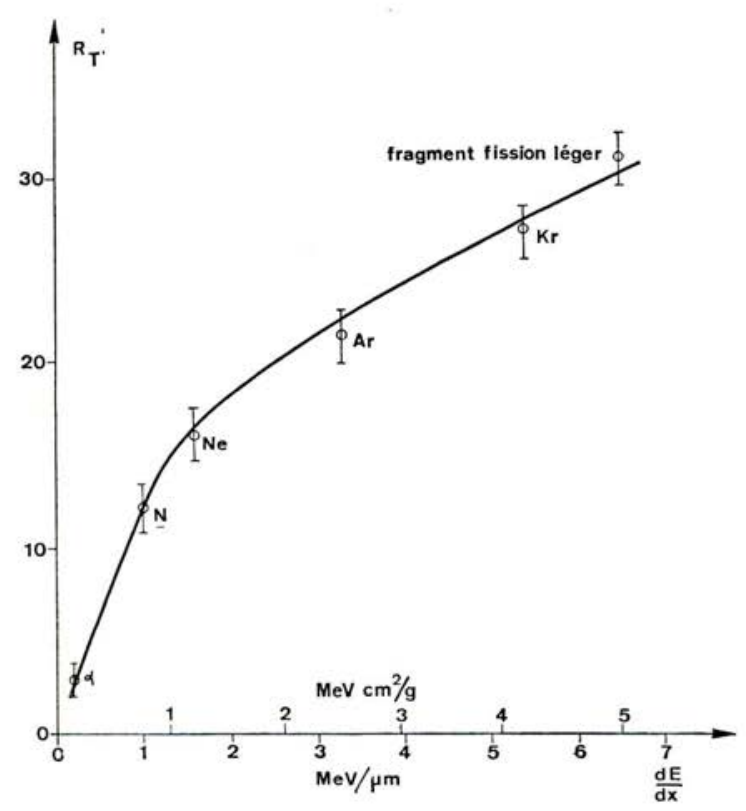

FIG. 5. - Variation de $R_{T}$ en fonction de la perte linéique d'énergie pour une solution de $\mathrm{KOH}(4 N)$ à $60^{\circ} \mathrm{C}$.

Bien qu'une certaine prudence s'impose, du fait que $\frac{\mathrm{d} E}{\mathrm{~d} x}$ n'est vraisemblablement pas, comme on le sait, le meilleur paramètre pour caractériser le comportement de la particule vis-à-vis du détecteur, on peut cependant avancer quelques hypothèses sur l'existence d'une valeur limite de $R_{T}$ :

- la première concerne le matériau : on peut supposer qu'il se sature, c'està-dire qu'il existe une quantité maximale de dommages dans une trace latente.

- la seconde met en cause l'agent chimique : la vitesse de diffusion de l'agent chimique dans la trace pourrait être inférieure à la vitesse $\bar{V}_{T}$ réelle,

\section{3 - Le rôle de L'Agent Chimique}

Nous avons fait varier la concentration et la température de la potasse et nous avons dans chaque cas mesuré $\mathrm{R}_{T}$ à partir de $\bar{V}_{g}$ et $\bar{V}_{T}$.

vOL. $7-\mathrm{N}^{\mathrm{O}} 4$ 
Ces vitesses obéissent à la loi d'ARrhenius, c'est-à-dire que, quelle que soit la concentration de l'agent chimique, ce sont des fonctions de la température absolue $T$ de la potasse de la forme :

$$
\begin{aligned}
& \bar{V}_{g}=A \exp \left(-\frac{W}{k T}\right) \\
& \bar{V}_{T}=B \exp \left(-\frac{W^{*}}{k T}\right)
\end{aligned}
$$

$W$ est l'énergie d'activation de la réaction potasse-matériau vierge. Nous avons trouvé : $W=0,95 \mathrm{eV}$.

$W^{*}$ est l'énergie d'activation de la réaction potasse-matériau endommagé. Les valeurs de $W^{*}$ pour différents ions sont consignées dans le tableau II.

TABLEAU II

QUelques VALEURS DE L'ÉNERGIE D'ACTIVATION W*

\begin{tabular}{|l|c|}
\hline \multicolumn{1}{|c|}{ Ion } & $\mathrm{W}^{*}(\mathrm{eV})$ \\
$\alpha(3 \mathrm{MeV})$ & 0,76 \\
$N(15,4 \mathrm{MeV})$ & 0,71 \\
$A(2 \mathrm{MeV})$ & 0,63 \\
Fragment de fission léger & 0,48 \\
Cf (100 MeV) & \\
\hline
\end{tabular}

$k$ est la constante de Boltzmann, $A$ et $B$ des constantes. Il en résulte que $\mathrm{R}_{T}$ suit lui aussi une loi exponentielle de la forme exp $\left(\frac{W-W^{*}}{k T}\right)$, c'est-à-
dire que :

- $R_{T}$ augmente lorsque la température diminue (tableau III). Un résultat analogue a été obtenu pour le nitrate de cellulose [s]. La trace révélée, de forme conique, présente un angle au sommet $\gamma$ tel que $\sin \gamma=\frac{\mathrm{I}}{\mathrm{R}_{T}}$; elle est plus fine,

TABLEAU III

VARIATION DE $R_{T}$ EN FONCTION DE LA TEMPÉRATURE DE L'AGENT CHIMIQUE POUR LES FRAGMENTS DE FISSION LÉGERS DU ${ }^{252}$ Cf ET UNE SOLUTION DE POTASSE

\begin{tabular}{|c|c|}
\hline $\begin{array}{l}\text { Température } \\
\text { (degré C) }\end{array}$ & $R_{T}$ \\
\hline 40 & 80 \\
\hline 50 & 62 \\
\hline 55 & 49 \\
\hline 60 & $3 I$ \\
\hline 70 & 23 \\
\hline
\end{tabular}
4 FOIS NORMALE A $60^{\circ} \mathrm{C}$ 
donc mieux repérable, lorsqu'on opère à basse température (c'est ce qu'on pourra faire en physique nucléaire pour repérer les dispositions relatives de certaines traces). Par contre, si l'on se borne à un décompte des traces, il vaut mieux accroître leurs dimensions, c'est-à-dire opérer à des températures plus élevées.

- $R_{T}$ augmente lorsque $W^{*}$ diminue. Nous avons vu plus haut que $R_{T}$ croît lorsque la particule est plus fortement ionisante. Les résultats donnés dans le tableau II sont donc cohérents : plus la particule est ionisante, plus le $W^{*}$ associé est faible.

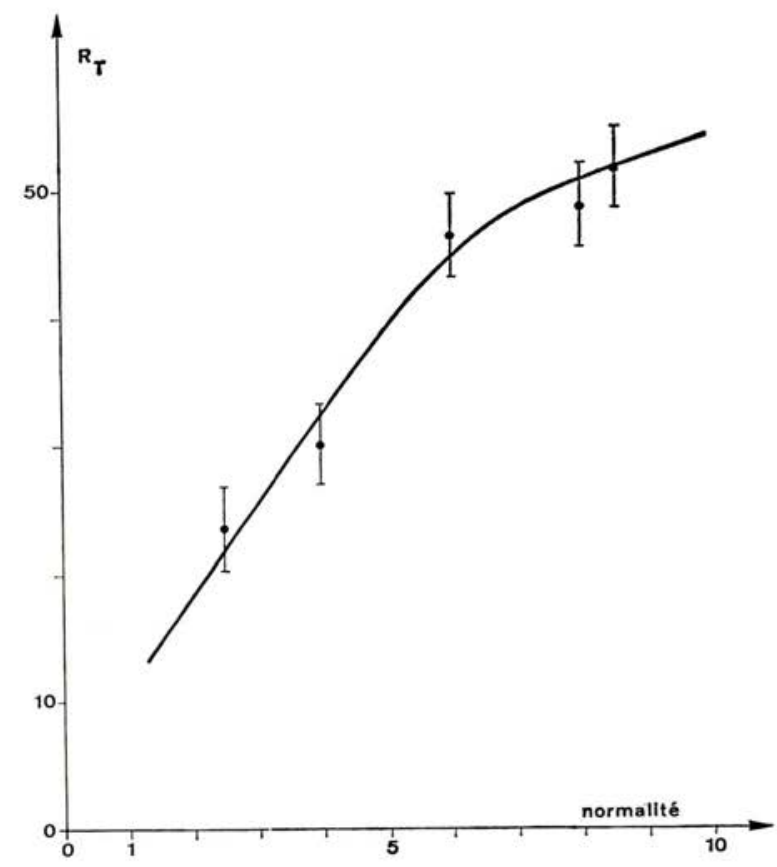

FIG. 6. - Variation de $\mathrm{R}_{T}$ en fonction de la normalité de la solution de potasse à $60^{\circ} \mathrm{C}$ pour les fragments de fission légers.

Lorsqu'on maintient la température de l'agent chimique à $60^{\circ} \mathrm{C}$ et que l'on fait varier sa concentration de $2 N$ à $, N, R_{T}$ augmente d'abord puis tend vers un palier (fig. 6) : la trace révélée est plus fine lorsque la concentration est plus élevée. Avec de la soude et des feuilles de triacétate de cellulose qu'il a préparées lui-même, Somogyr [6] a mis en évidence l'existence d'un maximum pour une concentration de $20 \%$. On peut penser que cette différence tient plus à la nature des matériaux employés qu'à celle de l'agent chimique. Nous avons constaté en effet qu'une solution de soude quatre fois normale à $60^{\circ} \mathrm{C}$ possède pratiquement le même rapport d'attaque (30,5 au lieu de $3 \mathrm{I}$ ) pour les fragments de fission légers du californium qu'une solution de potasse employée dans les mêmes

voL. 7 - $\mathrm{N}^{0} 4$ 
conditions. Par contre, le rôle joué par les constituants du matériau est très important; on en jugera à travers l'exemple qui est donné maintenant.

4 - Le rôle du plastifiant

Le triacétate de cellulose appelé triafol T.X. contient deux plastifiants :

- du phosphate de triphényle (environ is \%),

- du phtalate de dibutyle (environ, \%).

Pour les fragments de fission légers du californium et une solution de potasse quatre fois normale à $60^{\circ} \mathrm{C}$, son rapport d'attaque est de 82 au lieu de $3^{\mathrm{I}}$ pour le triafol T.N. La présence du plastifiant améliore considérablement les propriétés détectrices du matériau. Cela a incité notre laboratoire à fabriquer son propre triacétate de cellulose.

\section{III - L'ÉNERGIE MAXIMALE D'ENREGISTREMENT $E_{M}$}

$E_{M}$ est définie de la manière suivante : la trace d'une particule dont l'énergie est inférieure à $E_{M}$ est révélée en partie ou en totalité.

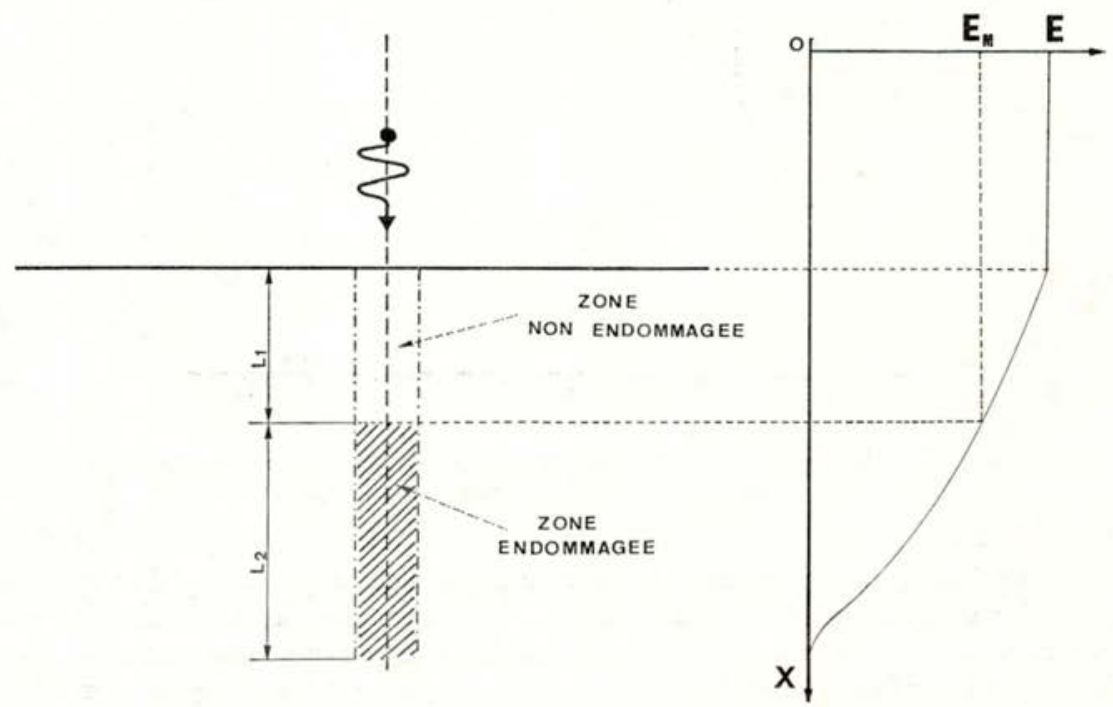

FIG. 7. - Définition de l'énergie maximale d'enregistrement.

Considérons par exemple une particule incidente d'énergie $E>E_{M}$ (fig. 7). $\mathrm{Au}$ cours de la traversée du matériau, son énergie diminue jusqu’à atteindre la valeur $E_{M}$ après une distance $l_{1}$ : les dommages créés sur cette première partie 
de la trajectoire sont insuffisants pour que la trace puisse être révélée. Au contraire, sur la partie restante de longueur $l_{2}$, la révélation est possible : $E_{M}$ dépend donc à la fois de la particule incidente et des conditions de l'attaque chimique. C'est un critère macroscopique mais qui est commode d'emploi.

Pour le déterminer, nous avons étudié la variation du temps $t_{r}=t_{a}-t_{v}$ en fonction de l'énergie de la particule incidente. En effet, le temps d'apparition des traces $t_{a}$ est la somme :

- du temps de développement $t_{v}$ nécessaire pour que le diamètre de la trace atteigne le seuil de visualisation que nous avons fixé à $0,5 \mu \mathrm{m}: t_{v}=\frac{0,5}{2 \bar{V}_{L E}}$ si $\bar{V}_{L E}$ est la vitesse d'élargissement moyenne de la trace d'une particule incidente d'énergie $E$.

- du temps $t_{r}$ nécessaire au rongement de l'épaisseur $l_{1}$ du matériau, pour que la particule n'ait plus qu'une énergie $E_{M}$.

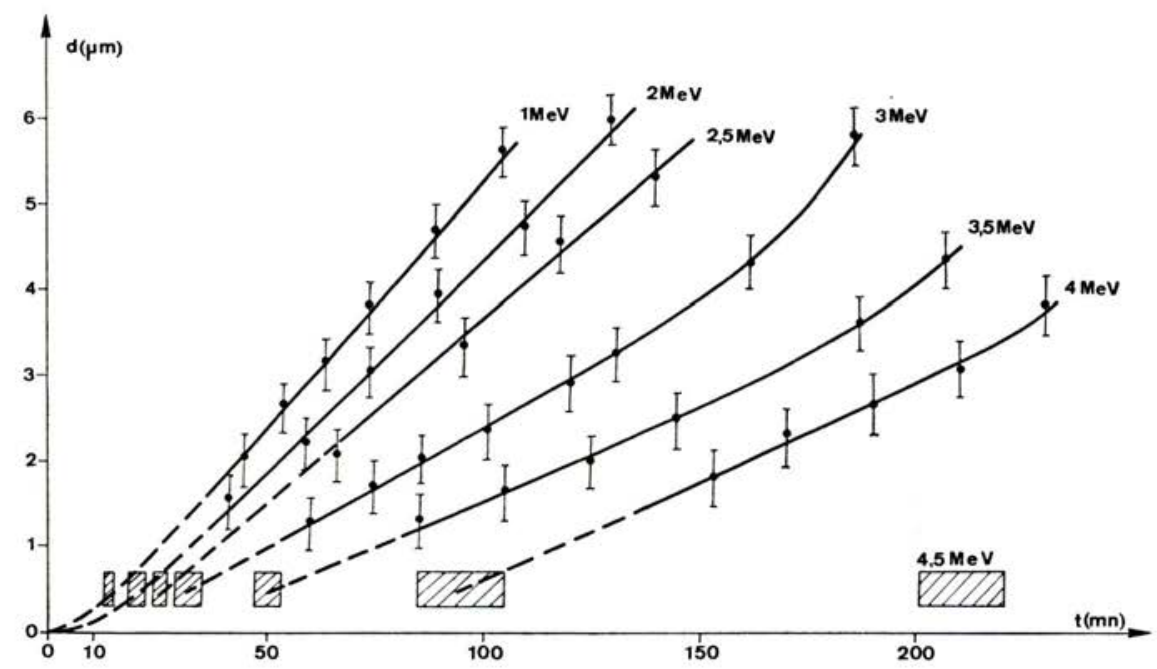

FIG. 8. - Variation du diamètre des traces de particules $\alpha$ d'incidence normale en fonction du temps de développement par une solution de $\mathrm{KOH}(4 \mathrm{~N})$ à $60^{\circ} \mathrm{C}$.

La mesure a été faite pour les particules $\alpha$ et pour une attaque chimique par de la potasse quatre fois normale à $60^{\circ} \mathrm{C}$. Il a fallu au préalable déterminer $t_{a}$ et $\bar{V}_{L E}$; la figure 8 donne les valeurs des diamètres $d$ des traces de particules $\alpha$ d'incidence normale selon le temps de développement $t$. L'instant d'apparition $t_{a}$ est représenté sous la forme d'un rectangle hachuré de façon à tenir compte des incertitudes sur le temps et le diamètre minimal observable. La vitesse d'élargissement des traces $\bar{V}_{L E}$ est égale à la moitié de la pente de la partie linéaire de la courbe $d(t)$ correspondant à l'énergie $E$.

vol. $7-\mathrm{N}^{\circ} 4$ 
La courbe $t_{r}(E)$, représentée sur la figure 9 , peut être divisée en trois régions :

- un palier entre $\circ$ et $3 \mathrm{MeV}$. Le fait que $t_{r}$ ne soit pas nul signifie sans doute que $\bar{V}_{L E}$ ne représente pas exactement la vitesse d'élargissement de la trace au début de l'attaque chimique; cela s'explique bien si l'on admet l'existence d'un temps d'incubation lorsque le processus chimique commence, comme l'a observé Debeauvais [9].

- une zone dite "de transition" entre 3 et $4 \mathrm{MeV}$ où $t_{r}$ se met à crôttre, - au-delà de $4 \mathrm{MeV}$, une zone où $t_{r}$ augmente fortement.

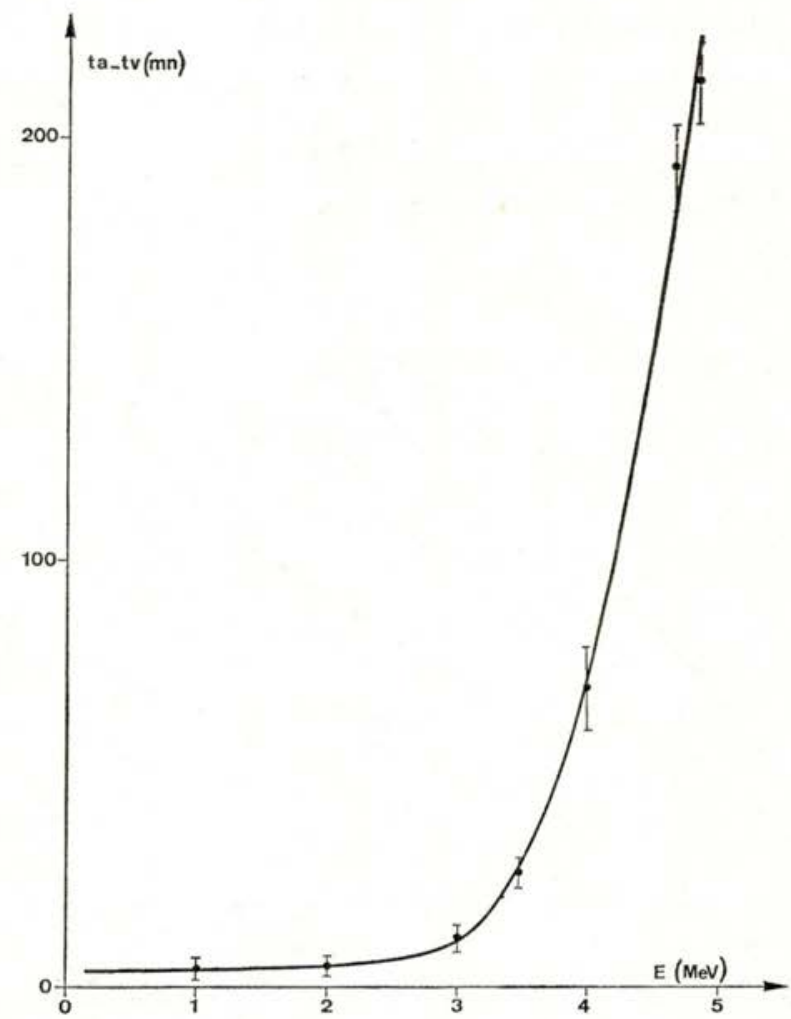

FIG. 9. - Variation de $t_{r}$ en fonction de l'énergie des particules $\alpha$.

Dans cette troisième région, la différence entre les temps $\left(t_{r}\right)_{2}$ et $\left(t_{r}\right)_{1}$ associés respectivement aux énergies $E_{2}$ et $E_{1}$ de la particule $\left(E_{2}>E_{1}\right)$ correspond, aux incertitudes expérimentales près, à la durée d'attaque chimique nécessaire pour enlever une couche de matériau dans laquelle l'énergie de la particule passe de $E_{2}$ à $E_{1}$. Ceci n'est plus vrai dans la zone de transition. C'est pourquoi, nous avons choisi pour valeur de $E_{M}, 4 \mathrm{MeV}$, qui est la limite supérieure de la zone de transition. 


\section{III - UN EXEMPLE D'APPLICATION \\ DU TRIACÉTATE DE CELLULOSE : LA SPECTROSCOPIE DES PARTICULES}

A partir des courbes de la figure 8, on peut établir une correspondance entre le diamètre $d$ des traces des particules $\alpha$ et leur énergie; c'est ce que nous avons représenté sur la figure 10. Au-dessus de $2 \mathrm{MeV}$, la relation est linéaire et il est possible de discriminer en énergie les particules avec une résolution d'environ soo $\mathrm{keV}$.

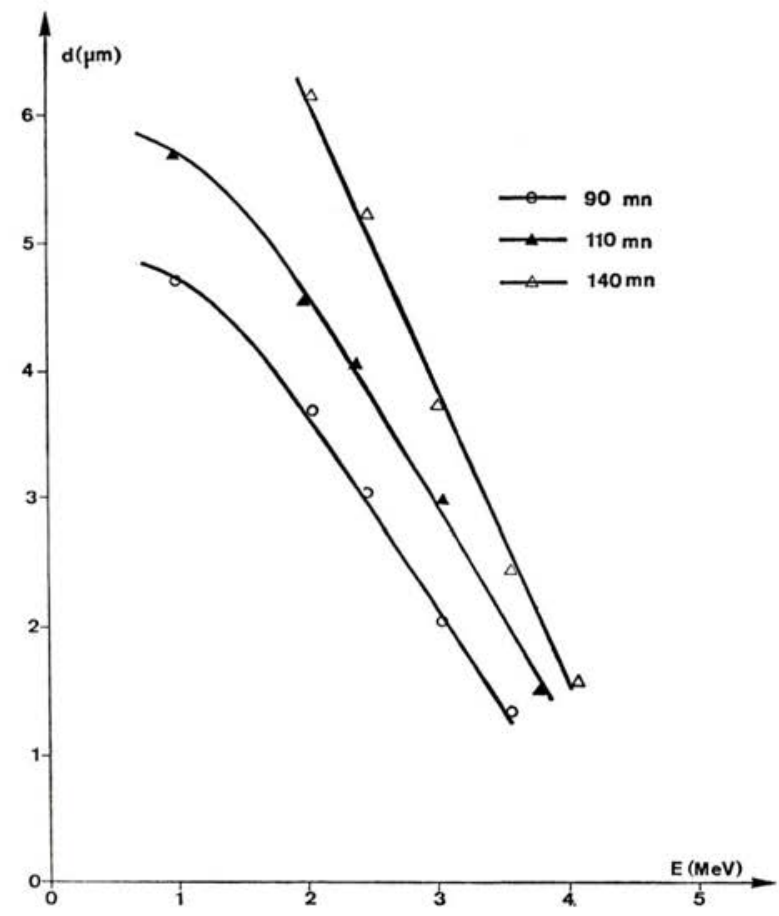

Fig. 10. - Variation du diamètre des traces de particules $\alpha$ en fonction de leur énergie pour différents temps de développement par $\mathrm{KOH}(4 \mathrm{~N})$ à $60^{\circ} \mathrm{C}$.

Une amélioration peut être obtenue en augmentant la durée de l'attaque chimique puisque la pente des droites $d(E)$ croît avec $t$. On a donc intérêt à prendre un temps de développement aussi grand que possible tout en restant compatible avec la gamme d'énergie explorée et une bonne définition de la trace. En effet, si le développement est trop prolongé, la trace " pâlit » et les mesures deviennent délicates.

Cette méthode, qui a déjà été utilisée avec différents matériaux [11] [1 12$]\left[\mathrm{I}_{3}\right]$ [14], devrait rendre de nombreux services dans le domaine de la spectroscopie.

vOL. 7 - No 4 


\section{BIBLIOGRAPHIE}

[I] Blanc D. Les Détecteurs Solides de Traces, Radioprotection : 1970, 5, I p. 37-78 - 1970, 5, 2, p. II 5-146 - 1970, 5, 3, p. 201-228.

[2] Monnin M. Les Détecteurs Solides de Traces, Radioprotection, 1967, 2, 2, p. 105-117.

[3] Chapuis A.M., Francors H., Nicomède-Gérard N. Contrôle de l'enrichissement de l'uranium en uranium 235 avec les traces laissées par les particules alpha dans le nitrate de cellulose, $1969,4,3$, p. 203-214.

[4] Vareille J.C. Thèse Doctorat de Spécialité, № 722, 1972, Limoges.

[s] Benton E.V. Thèse, 1968, Stanford.

[6] Somogyi G., Varnagy M., Medveczky L. The Influence of the etching parameters on the sensitivy of plastics, Rapport Conf. Int. Detecteurs Visuels Solides, 1969, ClermontFerrand III, p. 86-99.

[7] Blanford G.E., Fain J., Monnin M., Montret M., Touzery C. Calibration of plastic detectors for low energy cosmic rays. Rapport Int. Conf. Nuclear Photography. Solid State Track Detectors, Bucarest 1972.

[8] Blanford G.E. Thèse, 1971, Washington.

[9] Debeauvais M., Ralarosy J., RÉmy G., Strin R., Tripier J. Quelques applications récentes de nos connaissances expérimentales dans l'interaction des particules chargées avec les détecteurs plastiques. Quelques applications en découlant au Laboratoire de Physique Corpusculaire de Strasbourg. Rapport Conf. Int. Photographie Corpusculaire et Détecteurs Visuels Solides, 1970, Barcelone, I, p. 147-165.

[10] WeFEL J.P. Thèse, 1971, Washington.

[iI] Somogyi G., SchlenK B. The application of solide-state track detectors for measuring alpha particle angular distributions in nuclear reactions, Rapp. Conf. Int. Détecteurs Visuels Solides, Clermont-Ferrand, 1969, VIII, p. 27-40.

[12] Somogyr G. A New Method for measuring the particle energy according to the diameters of the holes in solid state nuclear track detectors, Atomki Kozlemenyek, 1966, 8, p. 21 8-224.

[13] Somogyi G., Schlenk B., Varnagy M. Mesko L., Valek A. Application of Solid state track detectors for measuring angular distributions of alpha particle groups from nuclear reactions, Nucl. Instr. and Meth., 1968, 63, p. 189-194.

[14] Meyerbrocker M. Alpha particles of natural radioactivity in air measured by cellulose nitrate plastic detectors, Rapport Conf. Int. Détecteurs Visuels Solides, ClermontFerrand, 1969, VI, p. s-10. 